\title{
A Micro-Bacterial Foraging Algorithm for High-Dimensional Optimization
}

\author{
Sambarta Dasgupta ${ }^{1}$, Arijit Biswas ${ }^{1}$, Swagatam Das ${ }^{1}$, Bijaya Ketan Panigrahi ${ }^{2}$ and Ajith Abraham ${ }^{3,4}$ \\ ${ }^{1}$ Department of Electronics and Telecommunication Engineering, Jadavpur University, Kolkata, India \\ swagatamdas19@yahoo.co.in \\ ${ }^{2}$ Department of Electrical Engineering, Indian Institute of Technology, Delhi, India \\ bkpanigrahi@ee.iitd.ac.in
}

${ }^{3}$ Center of Excellence for Quantifiable Quality of Service, Norwegian University of Science and Technology, Norway

${ }^{4}$ Machine Intelligence Research Labs - MIR Labs

ajith.abraham@ieee.org

\begin{abstract}
Very recently bacterial foraging has emerged as a powerful technique for solving optimization problems. In this paper, we introduce a micro-bacterial foraging optimization algorithm, which evolves with a very small population compared to its classical version. In this modified bacterial foraging algorithm, the best bacterium is kept unaltered, whereas the other population members are reinitialized. This new small population $\mu$-BFOA is tested over a number of numerical benchmark problems for high dimensions and we find this to outperform the normal bacterial foraging with a larger population as well as with a smaller population.
\end{abstract}

1. INTRODUCTION

In 2002 Kevin M Passino, proposed bacterial foraging as a tool for distributed optimization and control. This bacterial foraging optimization algorithm (BFOA) [1-4] mimics the foraging strategy of $E$. coli bacteria (those living in our intestines) which try to maximize the energy intake per unit time. From the very early days it has drawn attention of researchers due to its effectiveness in the optimization domain. So as to improve its performance a large number of modifications have already been undertaken. In $2007 \mathrm{Kim}$ et al. came up with BFOAGenetic algorithm (GA) hybridization for better performance [5]. In the same year authors in [6] proposed BFOA-Particle Swarm Optimization (PSO) hybridization, BFOA-Differential Evolution (DE) hybridization [7] and an adaptive bacterial foraging optimization (ABFOA) [8] for function optimization. But this micro bacterial foraging optimization is modeled specially for handling higher-dimensional problems, where the cost of computation becomes a major bottle-neck.

We took inspirations from similar approaches undertaken in GA and PSO. Initially $\mu$-GA [9] and $\mu$-PSO [10] also proved their efficiency over normal GA and PSO mainly for higher dimensional problems. We have tried to extend this concept for BFOA. The new version BFOA deals with a very small population size compared to normal BFOA, thus reducing the computational cost. Best bacterium in the swarm is kept unaltered after completion of iteration and others are scattered to some random locations following certain rules. This is done to main diversity in the population to avoid premature convergence.

We have tested the efficacy of this algorithm over a test bed which contains 5 high dimensional numerical benchmarks and it performs better than two versions of normal bacterial foraging one with population thirty and another with population three, same as the $\mu$ BFOA.

The rest of the paper is organized as follows. In Section 2, we briefly describe the bacterial foraging optimization algorithm. Section 3 introduces the modified version of bacterial foraging. Section 4 gives the computational results and illustrations. Finally the conclusion and future research scopes are enlisted in Section 5 .

II. The Classical Bacterial Foraging Optimization Algorithm

The bacterial foraging system consists of four principal mechanisms, namely chemotaxis, swarming, reproduction and elimination-dispersal [1]. A brief description of each of these processes along with the pseudo-code of the complete algorithm is described below.

i) Chemotaxis: This process simulates the movement of an E.coli cell through swimming and tumbling via flagella. Biologically an E.coli bacterium can move in two different ways. It can swim for a period of time in the same direction or it may tumble, and alternate between these two modes of operation for the entire lifetime. Suppose $\theta^{i}(j, k, l)$ represents i-th bacterium at $j$-th chemotactic, $k$-th reproductive and 1 -th 
elimination-dispersal step. C(i) is the size of the step taken in the random direction specified by the tumble (run length unit). Then in computational chemotaxis the movement of the bacterium may be represented by

$$
\theta^{i}(j+1, k, l)=\theta^{i}(j, k, l)+C(i) \frac{\Delta(i)}{\sqrt{\Delta^{T}(i) \Delta(i)}},
$$

where $\Delta$ indicates a vector in the random direction whose elements lie in $[-1,1]$.

ii) Swarming: An interesting group behavior has been observed for several motile species of bacteria including E.coli and S. typhimurium, where stable spatio-temporal patterns (swarms) are formed in semisolid nutrient medium. A group of E.coli cells arrange themselves in a traveling ring by moving up the nutrient gradient when placed amidst a semisolid matrix with a single nutrient chemoeffecter. The cells when stimulated by a high level of succinate, release an attractant aspertate, which helps them to aggregate into groups and thus move as concentric patterns of swarms with high bacterial density. The cell-to-cell signaling in E. coli swarm may be represented by the following function.

$$
\begin{aligned}
& J_{c c}(\theta, P(j, k, l))=\sum_{i=1}^{S} J_{c c}\left(\theta, \theta^{i}(j, k, l)\right) \\
& =\sum_{i=1}^{S}\left[-d_{\text {attractant }} \exp \left(-w_{\text {attractant }} \sum_{m=1}^{p}\left(\theta_{m}-\theta_{m}^{i}\right)^{2}\right)\right]+ \\
& \sum_{i=1}^{S}\left[h_{\text {repellant }} \exp \left(-w_{\text {repellant }} \sum_{m=1}^{p}\left(\theta_{m}-\theta_{m}^{i}\right)^{2}\right)\right]
\end{aligned}
$$

where $J_{c c}(\theta, P(j, k, l))$ is the objective function value to be added to the actual objective function (to be minimized) to present a time varying objective function, $S$ is the total number of bacteria, $p$ is the number of variables to be optimized, which are present in each bacterium and $\theta=\left[\theta_{1}, \theta_{2, \ldots \ldots \ldots \ldots . . .} \theta_{p}\right]^{T}$ is a point in the $p$ dimensional search domain. $d_{\text {aatractant }}, w_{\text {attractant }}, h_{\text {repellant }}, w_{\text {repellant }}$ are different coefficients that should be chosen properly.

iii) Reproduction: The least healthy bacteria eventually die while each of the healthier bacteria (those yielding lower value of the objective function) asexually split into two bacteria, which are then placed in the same location. This keeps the swarm size constant. iv) Elimination and Dispersal: Gradual or sudden changes in the local environment where a bacterium population lives may occur due to various reasons e.g. a significant local rise of temperature may kill a group of bacteria that are currently in a region with a high concentration of nutrient gradients. Events can take place in such a fashion that all the bacteria in a region are killed or a group is dispersed into a new location. To simulate this phenomenon in BFOA some bacteria are liquidated at random with a very small probability while the new replacements are randomly initialized over the search space.

III. THE MICRO - BFOA

A three bacteria population evolves through iterations in this proposed modification. We have used chemotactic operator for updating position of individual bacterium. In this algorithm the population size is very small. So, reproduction is not carried out to avoid saturation and premature convergence. Elimination dispersion step of original algorithm is adopted without any modification. After one complete chemotaxis loop, bacteria in population move to some new foraging locations. At this point, the population may be ranked according to objective function values. The best bacterium (rank 1) of the population retains its position. The second best bacterium (rank 2) is moved to a position very close to the best one. Worst bacterium (rank 3 ) is initialized at a random position. Fig.1 depicts a three bacteria system scattered over a single dimensional objective function landscape and steps undertaken after chemotaxis are shown. After each complete chemotaxis operation best bacterium contains most valuable information about the fitness landscape. So, by retaining its position we conserve the best discovered location. Now, second best bacterium is liquidated to a position close to the best bacterium in order to facilitate local search during next chemotactic loop execution. The worst bacterium is utilized in maintaining population diversity and avoiding premature convergence. Figure 2 provides a flow-chart for the algorithm. 


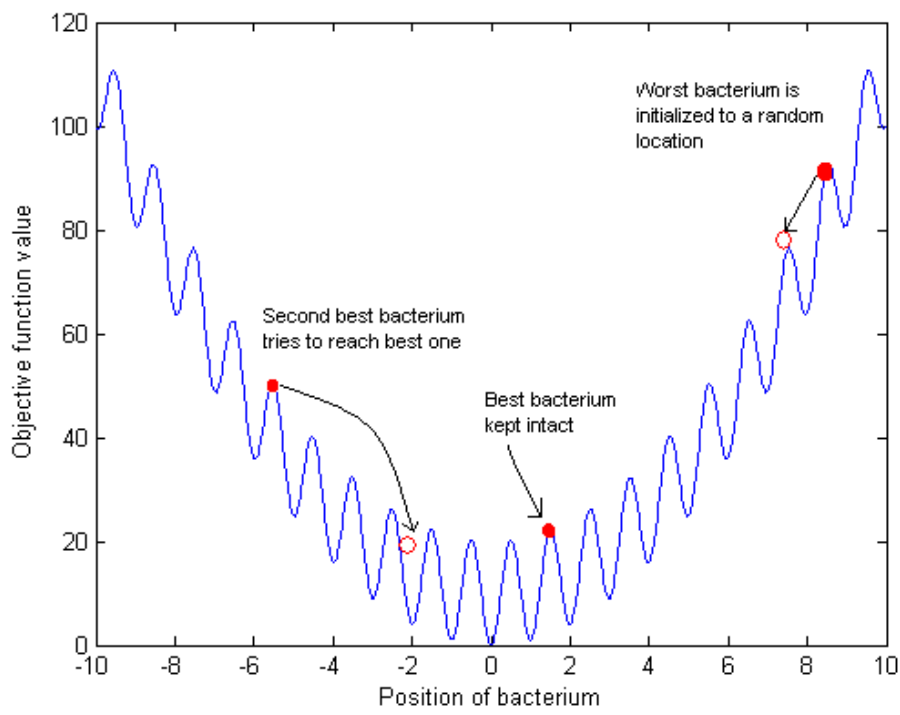

FIGURE 1: A SIMPLIFIED SCHEMATIC DIAGRAM FOR THREE BACTERIA MICRO SYSTEM OPTIMIZING SINGLE DIMENSIONAL OBJECTIVE FUNCTION.

\section{ANALYTICAL BASIS OF MICRO-BF}

Two main driving forces of the algorithm are exploration of entire functional landscape by worst bacterium and local search around best discovered location on fitness landscape. Let us denote bacteria triplet as $\vec{\theta}_{\text {best }}, \vec{\theta}_{\text {medium }}$ , $\vec{\theta}_{\text {worst }}$ according to their objective function value.

Theorem: Probability that $\vec{\theta}_{\text {worst }}$ will fall within the hypercube including $\vec{\theta}_{\text {best }}$ and $\vec{\theta}_{\text {medium }}$ decays exponentially with problem dimension.

Proof: At any instant of time in a particular dimension $p$

, $\max \left\|\vec{\theta}_{\text {best }}(p)-\vec{\theta}_{\text {medium }}(p)\right\|=\varepsilon$

Let us assume dimensionality of the optimization problem be $N$. Hence maximum volume of the hypercube including $\vec{\theta}_{\text {best }}$, and $\vec{\theta}_{\text {medium }}$ is $\mathcal{E}^{N}$.
Total volume of the hyperspace including entire search space is $(\max -\min )^{N}=$ Range $^{N}$.

Let, $P_{\text {saturation }}=$ Probability that $\vec{\theta}_{\text {worst }}$ will fall within the hypercube including $\vec{\theta}_{\text {best }}$ and $\vec{\theta}_{\text {medium }}$.

$\therefore P_{\text {saturation }}=$ Ratio of volume of two hyperspaces $=\frac{\varepsilon^{N}}{\text { Range }^{N}}=\left(\frac{\varepsilon}{\text { Range }}\right)^{N}$

Now, we have taken $\mathcal{E}=0.06 \times$ range

$\therefore P_{\text {saturation }}=0.06^{N}$

$\therefore P_{\text {saturation }} \rightarrow 0$ as $N$ becomes very large.

\section{Explanation:}

$P_{\text {saturation }}$ denotes the probability that $\vec{\theta}_{\text {worst }}$ will fall within the hypercube including $\vec{\theta}_{\text {best }}$ and $\vec{\theta}_{\text {medium }}$. In this case population diversity decreases and there is a chance for premature convergence. But probability of its occurrence is very small. In most of the time population diversity is maintained. So, the global search is almost never hampered. 


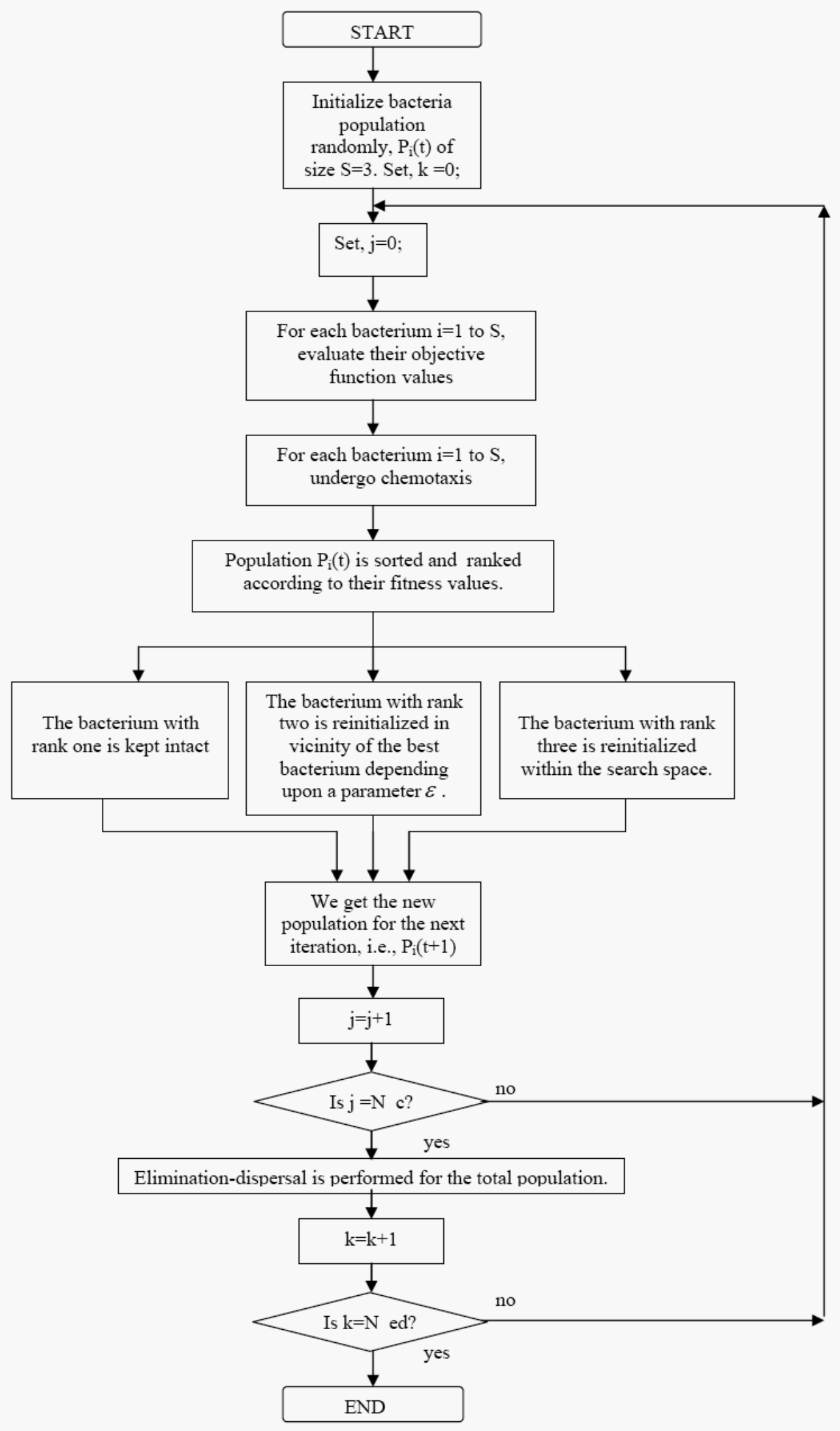

FIGURE 2: FLOW-CHART OF MICRO-BFOA. 
TABLE 1: DESCRIPTION OF BENCHMARK FUNCTIONS USED

\begin{tabular}{|c|c|c|c|}
\hline Function & Mathematical Representation & $\begin{array}{c}\text { Range of } \\
\text { search }\end{array}$ & Theoretical Optima \\
\hline $\begin{array}{l}\text { Ackley } \\
\left(f_{l}\right)\end{array}$ & $\begin{array}{l}f_{1}(\vec{X})=-20 \exp \left(-0.2 \sqrt{\frac{1}{500} \sum_{i=1}^{500} x_{i}^{2}}\right)- \\
\exp \left(\frac{1}{500} \sum_{i=1}^{500} \cos 2 \pi x_{i}\right)+20+e\end{array}$ & $(-2,2)^{500}$ & $f_{1}(\overrightarrow{0})=0$ \\
\hline $\begin{array}{l}\text { Griewank } \\
\qquad\left(f_{2}\right)\end{array}$ & $f_{2}(\vec{x})=\frac{1}{4000} \sum_{i=1}^{500} x_{i}^{2}-\prod_{i=1}^{500} \cos \left(\frac{x_{i}}{\sqrt{i}}\right)+1$ & $(-10,10)^{500}$ & $f_{2}(\overrightarrow{0})=0$ \\
\hline $\begin{array}{l}\text { Rastrigin } \\
\qquad\left(f_{3}\right)\end{array}$ & $f_{3}(\vec{x})=\sum_{i=1}^{500}\left[x_{i}^{2}-10 \cos \left(2 \pi x_{i}\right)+10\right]$ & $(-2,2)^{500}$ & $f_{3}(\overrightarrow{0})=0$ \\
\hline $\begin{array}{l}\text { Rosenbrock } \\
\qquad\left(f_{4}\right)\end{array}$ & $f_{4}(\vec{x})=\sum_{i=1}^{500-1}\left[100\left(x_{i+1}-x_{i}^{2}\right)^{2}+\left(x_{i}-1\right)^{2}\right]$ & $(-2,2)^{500}$ & $f_{4}(\overrightarrow{1})=0$ \\
\hline $\begin{array}{l}\text { Schwefel } \\
\qquad\left(f_{5}\right)\end{array}$ & $f_{5}(\vec{X})=-\sum_{i=1}^{500} x_{i} \sin \left(\sqrt{\left|x_{i}\right|}\right)$ & $(-500,500)^{500}$ & $f(\overrightarrow{416.99})=-416.99 \times 500$ \\
\hline
\end{tabular}

TABLE 2: PARAMETRIC SET-UP FOR $\mu$-BF

\begin{tabular}{|c|c|}
\hline Parameters & Parameter Values \\
\hline$S$ ( Population size $)$ & 3 \\
\hline$N_{-} c$ (Chemotactic loop counter $)$ & 50 \\
\hline$N_{-} e d($ elimination dispersal counter $)$ & 20 \\
\hline$p_{-} e d($ elimination dispersal probability $)$ & 0.25 \\
\hline $\mathcal{E}($ distance parameter $)$ & $0.06 \times$ range \\
\hline$C(i), i=1, \ldots . ., S$. (Step size $)$ & $0.2 \times$ range \\
\hline
\end{tabular}

\section{EXPERIMENTAL RESULTS}

To test the efficiency of the proposed micro bacterial foraging the following five numerical benchmark test functions have been chosen and are described in Table 1 . The dimension for all test problems have been taken to be 500.The parameters taken for the $\mu \mathrm{BFOA}$ algorithm is given in Table.2. In Table 2 range signifies difference between maximum and minimum value of the variable. The parameter $\varepsilon$ is the maximum separation introduced at a particular dimension between bacterium of rank 1 and
2 after chemotaxis. Step-size and $\mathcal{E}$ are scaled so that bacterium can have access to all parts of fitness landscape. We have compared the proposed algorithm with normal bacterial foraging with population size 30 and 3.For all these algorithms the maximum cut-off function evaluations is set to be 5000 .We take results for 50 independent runs and report the minimum ,mean and standard deviation of the final objective function values for the three competitive algorithms. Performance is illustrated in Figure 3. 
TABLE 3: CONVERGENCE DATA FOR FIVE BENCHMARK FUNCTIONS

\begin{tabular}{|c|c|c|c|c|}
\hline $\begin{array}{c}\text { Name } \\
\text { of } \\
\text { function }\end{array}$ & $\begin{array}{c}\text { Statistical } \\
\text { Measurements }\end{array}$ & $\begin{array}{c}\text { Micro Bacterial } \\
\text { Foraging }(\mathrm{pop}=3)\end{array}$ & $\begin{array}{c}\text { Bacterial } \\
\text { Foraging }(\text { pop }=30)\end{array}$ & $\begin{array}{c}\text { Bacterial } \\
\text { Foraging }(\text { pop }=3)\end{array}$ \\
\hline \multirow{3}{*}{ Ackley } & Minimum & 3.5924 & 4.4981 & 4.0712 \\
\hline & Mean & 3.8175 & 4.6555 & 4.3383 \\
\hline & Std. dev. & 0.1128 & 0.0583 & 0.1549 \\
\hline \multirow{3}{*}{ Griewank } & Minimum & 2.5401 & 3.5872 & 3.6338 \\
\hline & Mean & 2.7074 & 3.7690 & 3.7693 \\
\hline & Std. dev. & 0.0394 & 0.0426 & 0.0459 \\
\hline \multirow{3}{*}{ Rastrigin } & Minimum & 2151.2082 & 3077.7121 & 2892.6204 \\
\hline & Mean & 2285.0032 & 3052.8201 & 3102.7995 \\
\hline & Std. dev. & 36.5299 & 27.6755 & 56.7978 \\
\hline \multirow{3}{*}{ Rosenbrock } & Minimum & $\mathbf{4 5 7 4 8 . 2 5 7 0}$ & 62805.2019 & 67669.1890 \\
\hline & Mean & 49035.5738 & 66463.91399 & 71454.2668 \\
\hline & Std. dev. & 1745.5252 & 777.7251 & 884.7100 \\
\hline \multirow{3}{*}{ Schwefel } & Minimum & -96558.5871 & -98661.0729 & -97528.9802 \\
\hline & Mean & -93079.6066 & -93905.7658 & -92945.7018 \\
\hline & Std. dev. & 2067.9320 & 1904.1674 & 1905.7137 \\
\hline
\end{tabular}

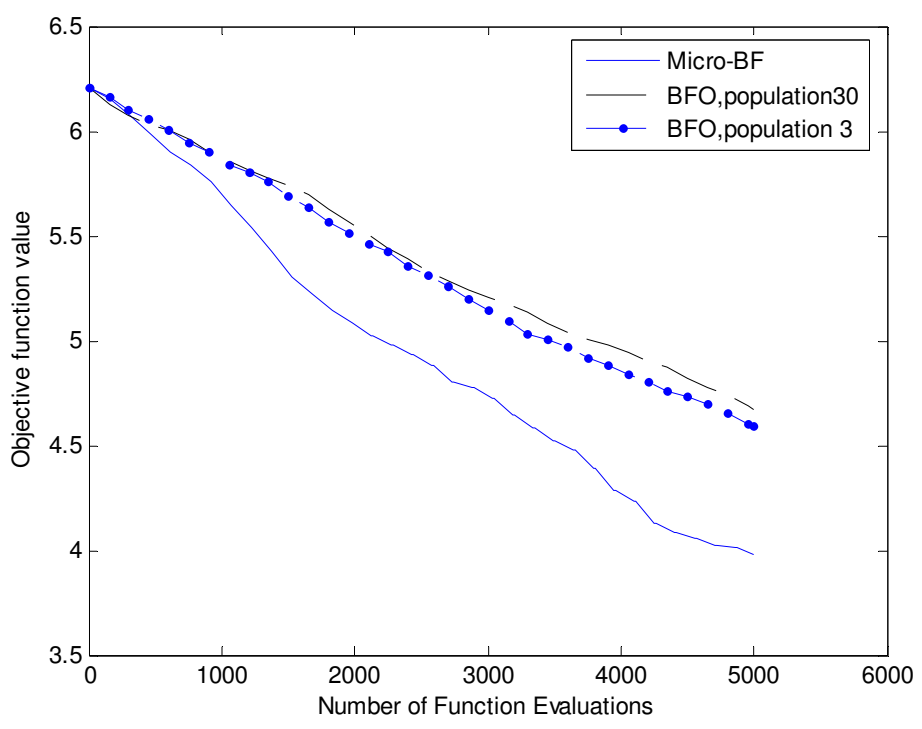

(a)

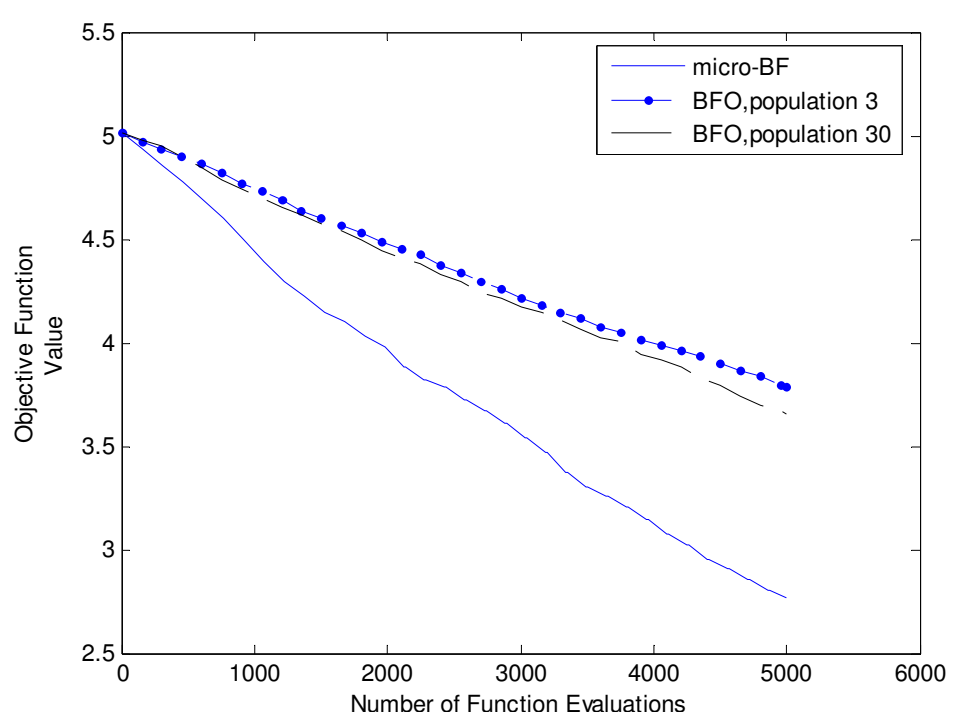

(b) 


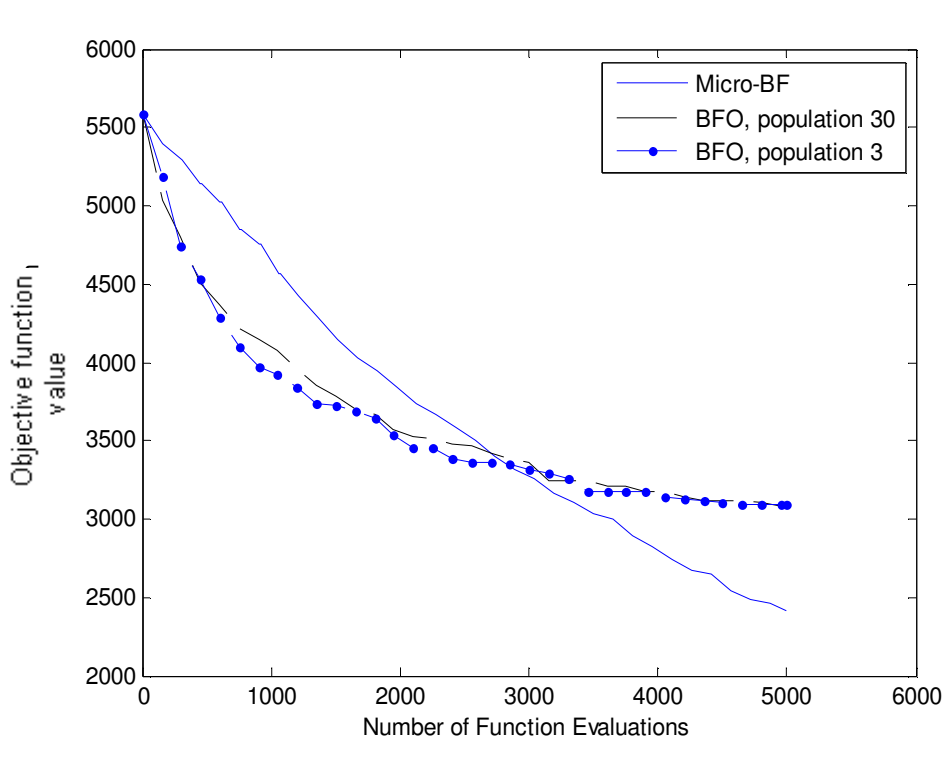

(c)

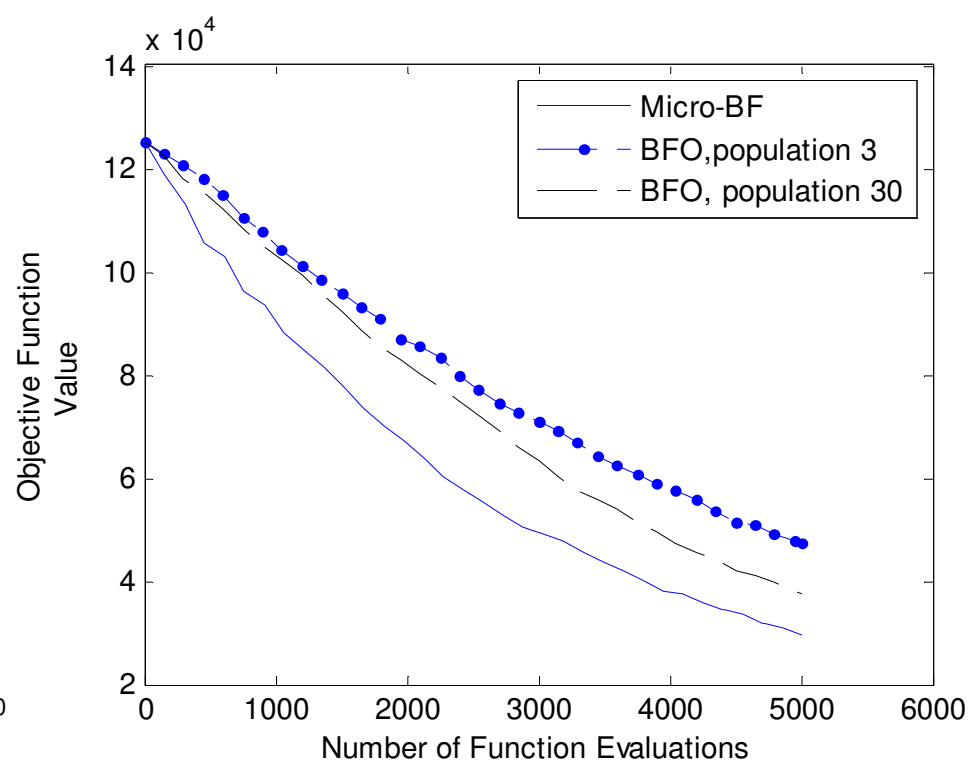

(d)

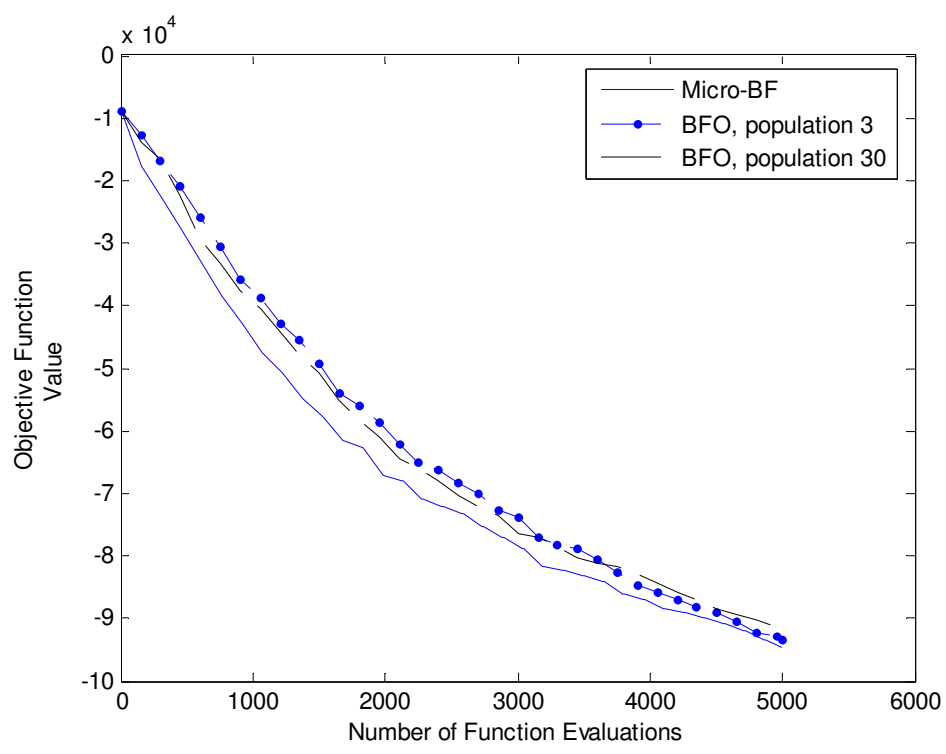

(e)

FigURE 3: A COMPARATIVE STUDY OF MICRO-BF WITH CLASSICAL BFO OVER VARIOUS FUNCTIONS FOR DIMENSION=500. ACKLEY FUNCTION, (B) GRIEWANGK FUNCTION, (C) RASTRIGIN FUNCTION, (D) ROSENBROCK FUNCTION, AND (E) SCHWEFEL FUNCTION.

\section{CONCLUSIONS}

This paper presents a new version of bacterial foraging optimization which evolves with a very small population of size three and designed specially for tackling higher dimensional problems. The major advantage of this algorithm lies in the reduction of computational cost and computational time. The newly developed algorithm is highly suitable for real time computing. Performance of the proposed algorithm is tested on a test bed of five benchmark functions where it performed well enough compared to the normal bacterial foraging with population size thirty and with population size three. Further research work may focus on developing some new algorithms related to bacterial foraging to decrease the computational cost and time during global optimization. Also some modifications of the normal bacterial foraging 
optimization can be undertaken to enhance the performance of the said algorithm near global optima.

REFERENCES

[1]. K. M. Passino, "Biomimicry of bacterial foraging for distributed optimization and control," IEEE. Control Syst. Mag., pp. 52-67, June. 2002.

[2]. Liu, Y., and Passino, K.M., Biomimicry of Social Foraging Bacteria for Distributed Optimization Models, Principles, and Emergent Behaviors, Journal of Optimization Theory and Applications, Vol. 115, no3, pp. 603-628, Dec. 2002.

[3]. V. Gazi and K. M. Passino, "Stability analysis of swarms in an environment with an attractant/repellent profile," in Proc. American Control Conf., Anchorage, Alaska, May 2002, pp. 1819-1824.

[4]. V. Gazi and K. M. Passino, "Stability analysis of swarms," in Proc. American Control Conf., Anchorage, Alaska, May 2002, pp. 1813-1818.

[5]. Kim, D. H., Abraham, A., Cho, J. H.: A hybrid genetic algorithm and bacterial foraging approach for global optimization, Information Sciences, Vol. 177 (18), 3918-3937, (2007).

[6]. A. Biswas ,S. Dasgupta, S Das, and A. Abraham,: Synergy of PSO and Bacterial Foraging Optimization: A Comparative Study on Numerical Benchmarks, Second International Symposium on Hybrid Artificial Intelligent Systems (HAIS 2007), Advances in Soft computing Series, Springer Verlag, Germany, E. Corchado et al. (Eds.): Innovations in Hybrid Intelligent Systems, ASC 44, pp. 255-263, (2007).

[7]. Arijit Biswas, Sambarta Dasgupta, Swagatam Das, and Ajith Abraham, A Synergy of Differential Evolution and Bacterial Foraging Algorithm for Global Optimization, International Journal on Neural and Mass-Parallel Computing and Information Systems, Neural Network World, Volume 17, No. 6, pp. 607-626, 2007.

[8]. S. Dasgupta, S Das, A. Abraham, and A. Biswas, Adaptive Computational Chemotaxis in Bacterial Foraging Optimization: an Analysis, IEEE Transactions on Evolutionary Computing, (in press), 2008.

[9]. D. E. Goldberg, "Sizing populations for serial and parallel genetic algorithms", in Proc. 3rd International Conference Genetic Algorithms (ICGA 89), 1989, pp.70-79.]

[10]. T Huang and A. S. Mohan, "Micro-particle swarm optimizer for solving higher dimensional problems
( $\mu$ PSO for high dimensional problems)", Applied Mathematics and Computation 181 (2006) 1148-1154, Science Direct. 